\title{
Mutational analysis of the major proline transporter (PrnB) of Aspergillus nidulans
}

\section{S. N. Tavoularis, U. H. Tazebay, G. Diallinas, M. Sideridou, A. Rosa, C. Scazzocchio \& V. Sophianopoulou}

To cite this article: S. N. Tavoularis, U. H. Tazebay, G. Diallinas, M. Sideridou, A. Rosa, C. Scazzocchio \& V. Sophianopoulou (2003) Mutational analysis of the major proline transporter (PrnB) of Aspergillus nidulans, Molecular Membrane Biology, 20:4, 285-297, DOI: 10.1080/0968768031000106339

To link to this article: https://doi.org/10.1080/0968768031000106339

曲 Published online: 09 Jul 2009.

Submit your article to this journal $₫$

山 Article views: 36

Citing articles: 13 View citing articles 


\section{Mutational analysis of the major proline transporter (PrnB) of Aspergillus nidulans}

\author{
S. N. Tavoularis $\uparrow$, U. H. Tazebay $\S$, G. Diallinas $\uparrow$, \\ M. Sideridou†, A. Rosa\#, C. Scazzocchioł¥ and \\ V. Sophianopoulou ${ }^{*}$
}

$\dagger$ Institute of Biology, National Center for Scientific Research 'Demokritos' (NCSRD), Aghia Paraskevi 15310 Athens, Greece

* Institut de Génétique et Microbiologie, UMR CNRS C8621, Université de Paris-XI, Centre d'Orsay, F-91405 Orsay Cedex, France

$\S$ Department of Molecular Biology and Genetics, Bilkent University, Bilkent 06533 Ankara, Turkey

- Faculty of Biology, Department of Botany, University of Athens, Panepistimioupolis, Athens 157 81, Greece

\# Instituto de Investigación Médica, Mercedes y Martín Ferreyra (INIMEC-CONICET), Córdoba, Argentina

$¥$ Institut Universitaire de France, France

\begin{abstract}
Summary
PrnB, the L-proline transporter of Aspergillus nidulans, belongs to the Amino acid Polyamine Organocation (APC) transporter family conserved in prokaryotes and eukaryotes. In silico analysis and limited biochemical evidence suggest that APC transporters comprise 12 transmembrane segments (TMS) connected with relatively short hydrophilic loops (L). However, very little is known on the structure-function relationships in APC transporters. This work makes use of the A. nidulans PrnB transporter to address structure-function relationships by selecting, constructing and analysing several prnB mutations. In the sample, most isolated missense mutations affecting PrnB function map in the borders of cytoplasmic loops with transmembrane domains. These are I119N and G120W in L2-TMS3, F278V in L6-TMS7, NRT378NRTNRT and PY382PYPY in L8TMS9 and T456N in L10-TMS11. A single mutation (G403E) causing, however, a very weak phenotype, maps in the borders of an extracellular loop (L9-TMS10). An important role of helix TMS6 for proline binding and transport is supported by mutations K245L and, especially, F248L that clearly affect PrnB uptake kinetics. The critical role of these residues in proline binding and transport is further shown by constructing and analysing isogenic strains expressing selected prnB alleles fused to the gene encoding the Green Fluorescent Protein (GFP). It is shown that, while some prnB mutations affect proper translocation of PrnB in the membrane, at least two mutants, K245E and F248L, exhibit physiological cellular expression of PrnB and, thus, the corresponding mutations can be classified as mutations directly affecting proline binding and/or transport. Finally, comparison of these results with analogous studies strengthens conclusions concerning amino acid residues critical for function in APC transporters.
\end{abstract}

*To whom correspondence should be addressed. e-mail: vicky@bio.demokritos.gr
Keywords: Aspergillus nidulans, proline, transport, mutagenesis, structure-function analysis.

\begin{abstract}
Abbreviations: TMS, transmembrane segment, L, hydrophilic loop, CAR, Consensus Amphipathic Region, APC, Amino acid Polyamine Organocation transporter family, GFP, Green Fluorescent Protein, $\mathrm{MM}$ and $\mathrm{CM}$, minimal and complete media respectively, $K_{\mathrm{m}}$, Michaelis constant, $V_{\max }$, maximum velocity of transport.
\end{abstract}

\section{Introduction}

Amino acids and their derivatives are transported into and out of cells by a variety of transporters which comprise several distinct protein families, some of which are distantly related (Saier 2000). The largest and best-studied amino acid transporter family is the Amino acid Polyamine Organocation (APC) transporter family (Sophianopoulou and Diallinas 1995, Saier 2000). The APC transporter superfamily includes members that function as solute/cation symporters and solute/solute anti-porters. They are found in bacteria, archaea, fungi, eukaryotic protists, plants and animals. They vary in length, from 350-850 residues. Most of them possess 12 transmembrane $\alpha$-helical segments (TMS), but members of some sub-families might have 10 , 11 or 14 TMS. One APC family member, Hip1p of Saccharomyces cerevisiae has been implicated in heavy metal transport (Farcasanu et al. 1998). Interestingly, three integral membrane receptors of mammals, the ecotropic retroviral leukaemia receptor (ERR), the human retroviral receptor (HRR) and the T-cell early activator $\left(T_{\text {ea }}\right)$, are homologous to APC transporters (Reizer et al. 1993). The ERR protein has been shown to function as a cation/amino acid co-transporter (Wang et al. 1991). Other proteins, including the developmentally controlled GerAll spore germination protein of Bacillus subtilis and the acetylcholine receptor of Drosophila melanogaster might also share a common evolutionary origin with members of the APC family (Reizer et al. 1993).

Several APC bacterial and fungal amino acid transporters have been identified and studied in great detail at the level of transport kinetics and regulation of expression (Sophianopoulou and Diallinas 1995, Saier 2000, Burkovski and Kramer 2002 and references therein). Fungal and bacterial amino acid transporters show significant sequence similarities (33-62\% identity scores in binary comparisons) that may reflect a common topology and mechanism of action. Their specificities range from one to several L-amino acids and their kinetics of transport and regulation of expression may also vary significantly (Sophianopoulou and Diallinas 1995, Saier 2000). Studies addressing how yeast amino acid transporters find their way to the plasma membrane have shown that their topogenesis depends on both general and family-specific secretion factors or chaperones (Martinez and 
Ljungdahl 2000). Finally, recent studies have shown that homologues of amino acid transporters in yeast function as 'sensors' of amino acids rather than real transporters (Bernard and André 2001, Forsberg and Ljungdahl 2001).

In Aspergillus nidulans, the prnB gene (Sophianopoulou and Scazzocchio 1989) encodes a highly specific transporter for L-proline (Sophianopoulou and Diallinas 1995, Tazebay et al. 1995). The prnB gene is located in the prn gene cluster encoding all proteins necessary for proline catabolism (Hull et al. 1989). Expression of the prnB gene has been studied in great detail and shown to be induced by proline, amino acid starvation and conidial germination and to be repressed by the simultaneous presence of ammonia and glucose (Arst and Cove 1973, Sophianopoulou et al. 1993, Tazebay et al. 1995, Gonzalez et al. 1997, Tazebay et al. 1997, Cubero et al. 2000).

This work makes use of the $A$. nidulans PrnB transporter to address structure-function relationships by selecting, constructing and analysing several prnB mutations. Studying chimeric proteins carrying PrnB mutations fused to the green fluorescent protein (GFP) allowed one to classify several mutations to those affecting PrnB topogenesis and to those directly affecting proline binding and transport. The results also showed that several of the amino acids affecting PrnB function are located in similar regions with residues affecting the function of homologous APC transporters from bacteria, yeast and mammals.

\section{Results and discussion}

\section{Genetic isolation of prnB mutations}

Early genetic work has led to the isolation of several prnBspecific loss-of-function mutations (prnB6, prnB32, prnB36, prnB81, prnB82, prnB1110, prnB206; Arst and MacDonald 1975, Arst et al. 1981, Jones et al. 1981, Arst Jr., H. N., personal communication). A detailed genetic map including most of these mutations has been constructed and correlated with the physical map available for the prnB locus (Durrens et al. 1986). To enlarge the original collection of prnB mutations and gain further insights into the process of PrnB structure and function, the original genetic selection was modified in order to isolate partial loss-of-function or cryosensitive mutations (see Experimental procedures). Such mutations are more likely to affect PrnB function rather than its expression. One, thus, isolated six novel prnB mutations called prnB115, prnB117, prnB119, prnB144, prnB411 and prnB508.

All prnB mutants isolated previously and herein were analysed by simple growth tests for their ability to grow on proline or other nitrogen sources. Figure 1 shows selected results for growth on proline at 25 and $37^{\circ} \mathrm{C}$. Within the limits of growth tests, two types of mutants could be distinguished. Type 1 mutants, which included all the unconditional mutations from the original collection of Arst et al . (1981), showed a growth phenotype identical to that of the total loss-offunction mutation prnB377 resulting from an internal deletion in the prnB gene (Tazebay et al. 1995, 1997). Type 2 mutants (prnB115, prnB119, prnB144, prnB411, prnB117 and prnB508) showed a growth phenotype compatible, albeit at different degrees, with a partial loss of PrnB function, more evident at $25^{\circ} \mathrm{C}$ than at $37^{\circ} \mathrm{C}$. None of the mutations resulted in a $\mathrm{pH}$-dependent phenotype and none affected growth on other nitrogen sources tested (results not shown).

\section{Sequence changes of prnB mutations}

The entire $p r n B$ open reading frame of each of the mutants described above was sequenced. The region between the start and stop codons of prnB was amplified by the polymerase chain (PCR) reaction, as described in Experimental procedures. PCR products were either sequenced directly or cloned into bluescript $\mathrm{KS}(+)$ and sequenced using a series of prnB-specific oligonucleotides (see Experimental procedures). Unique mutations were found in every case. The substitutions identified are summarized in Table 1. Among the 13 mutations sequenced, four (prnB32, prnB36, prnB82 and prnB1110) corresponded to nonsense and/or frameshift mutations and were not analysed any further in this work. The physical location of the mutations that have been mapped previously (Jones et al. 1981) is completely congruent with their location in the genetic fine structure map (results not shown, Durrens et al. 1986). All isolated mutations studied further were renamed on the basis of the corresponding amino acid substitution (see Table 1).

\section{A mutation affecting prnB mRNA steady state levels}

To rule out the possibility that isolated mutations affect prnB expression rather than PrnB function, it was investigated whether basal prnB mRNA steady state levels are comparable to wild-type levels. Northern blot analyses were carried out of all prnB mutations at 25 and $37^{\circ} \mathrm{C}$. It was found that all mutant strains, except prnB-Extended (prnB115), showed mRNA levels identical to those of a prnB ${ }^{+}$strain at both temperatures (results not shown). Mutant prnB-Extended (prnB115) showed reduced prnB mRNA at $25^{\circ} \mathrm{C}$ (Figure $2(a))$. This mutation is due to a frameshift mutation in the physiological stop codon of the prnB gene, resulting in a seven amino acids extension of the PrnB open reading frame. Interestingly, the region of the prnB mRNA immediately downstream from the stop codon contains sequences which might form a stem-loop structure (Figure 2(b)). In some cases, such structures have been shown to be critical for mRNA stability and turnover (Causton et al. 1994, Platt et al. 1996). Thus, one can speculate that extended translation of this region might well disrupt the formation of this stemloop secondary structure and, thus, affect prnB mRNA steady state levels.

\section{Design and construction of site-directed prnB mutations}

A second approach to identify residues important for PrnB function or specificity was to construct by in vitro directed mutagenesis missense mutations altering selected amino acids conserved in the proline transporters of $A$. nidulans (PrnB) and Saccharomyces cerevisiae (Put4p). The mutations introduced concern two specific amino acid residues, Q219 located in L5 and K245 located within TMS6 of the 


\section{Uric Acid}
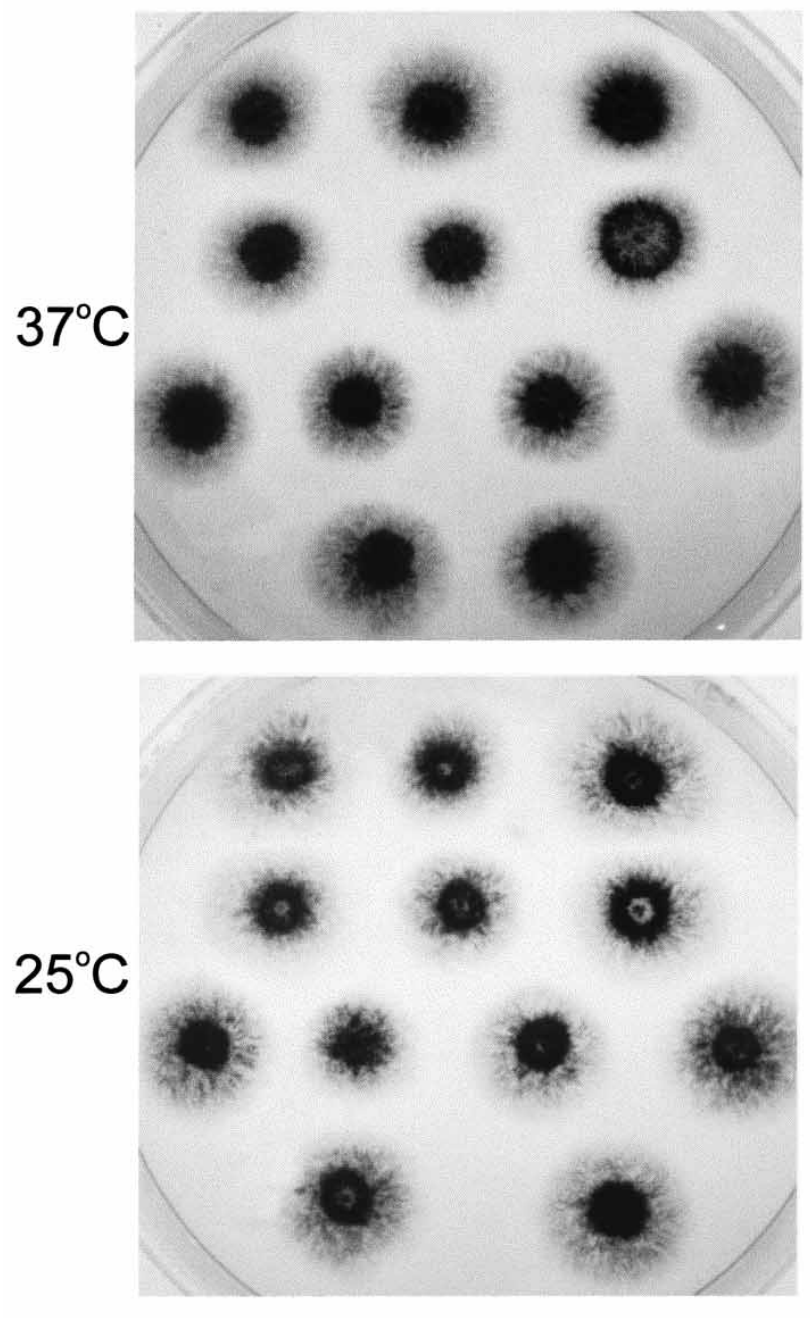

\section{Proline}
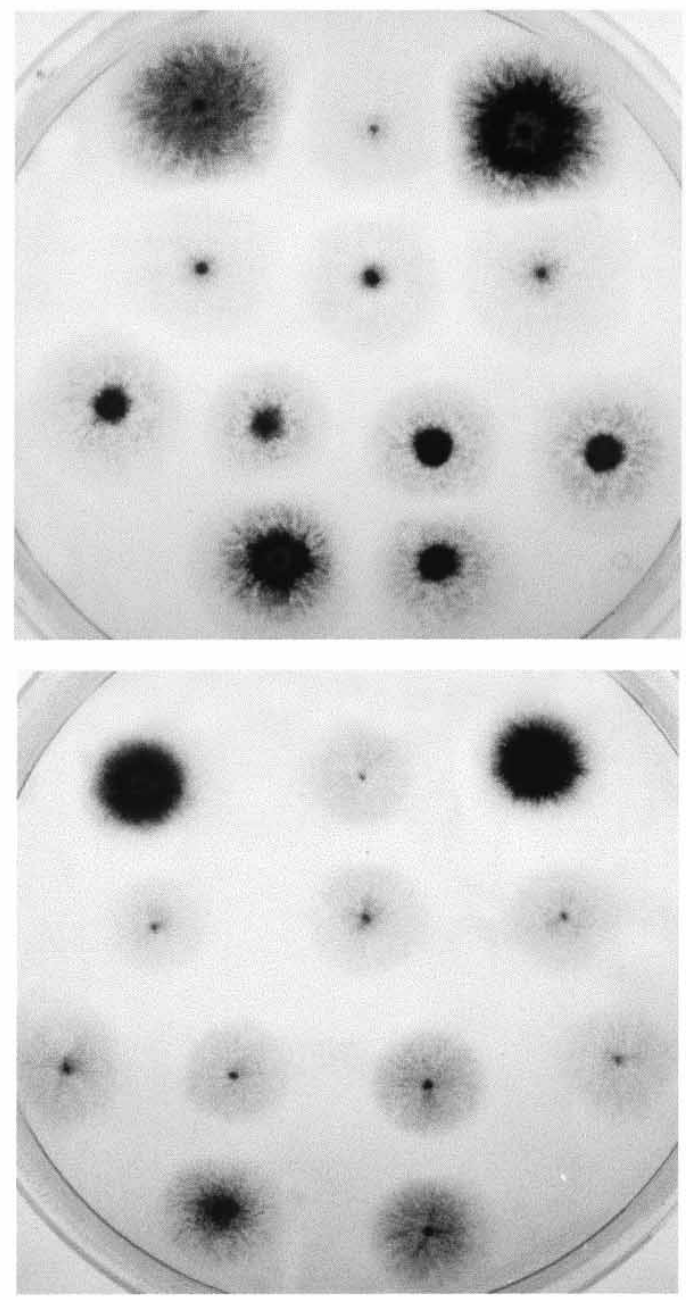

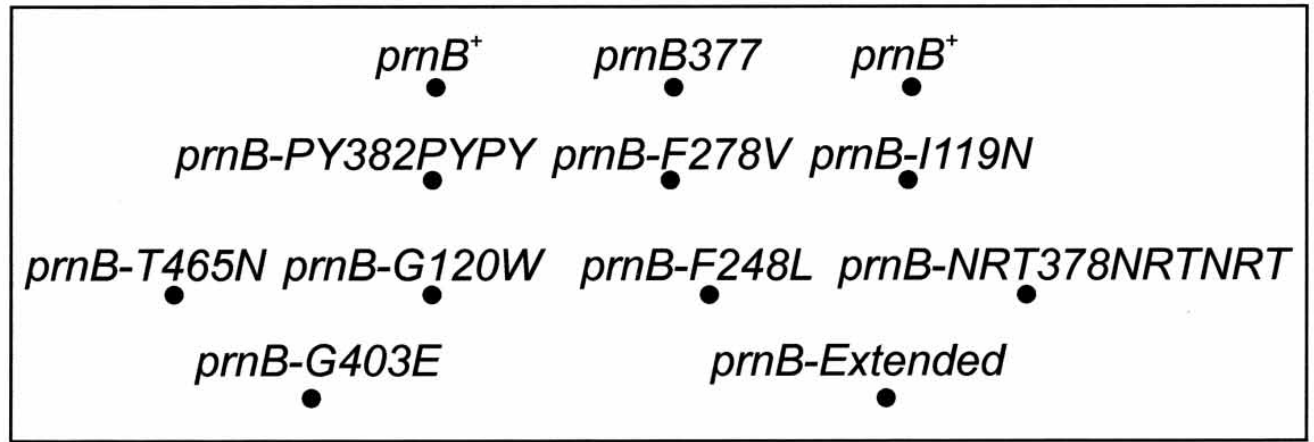

Figure 1. Growth of $A$. nidulans mutant strains. Growth of control strains (prnB ${ }^{+}$, prnB377) and mutant strains (prnB-PY382PYPY, prnBF278V, prnB-l119N, prnB-T456N, prnB-G120W, prnB-F248L, prnB-NRT378NRTNRT, prnB-G403E and prnB-Extended). Because prnB mutations exist in either green $\left(y A^{+}\right)$or yellow ( $\left.y A 2\right)$ conidiospore genetic background, the corresponding prn $B^{+}$strains are shown. Growth tests were carried out on $A$. nidulans minimal medium supplemented with $595 \mu \mathrm{M}$ uric acid or $5 \mathrm{mM}$ proline as sole nitrogen sources at 25 and $37^{\circ} \mathrm{C}$, as indicated. Growth tests were carried out for $60 \mathrm{~h}$ at $25^{\circ} \mathrm{C}$ or $48 \mathrm{~h}$ at $37^{\circ} \mathrm{C}$.

PrnB protein. The choice was based on a number of observations. Both residues are located in segments of high conservation in fungal transporters (Figure 3). In addition, K245 is one of the two positively charged residues located within a TMS of PrnB. The other is H334 located within TMS8. Interestingly, a Lys residue in TMS5 of the phenylalanine transporter (PheP) of $E$. coli has been shown to be critical for transport activity (Pi et al. 1993). Charged or polar amino acid residues in general might be involved directly or indirectly in protein function. 
Table 1. Mutant alleles of $p r n B$ and their phenotypes on proline as sole nitrogen source.

\begin{tabular}{|c|c|c|c|c|c|}
\hline \multirow[b]{2}{*}{ prnB allele } & \multicolumn{2}{|c|}{ Phenotype on proline } & \multirow[b]{2}{*}{ Nucleotide change } & \multirow[b]{2}{*}{ Protein change } & \multirow[b]{2}{*}{ L-TMS } \\
\hline & $25^{\circ} \mathrm{C}$ & $37^{\circ} \mathrm{C}$ & & & \\
\hline$p r n B^{+}$ & ++++ & ++++ & - & - & - \\
\hline prnB377 & - & & Deletion (Tazebay et al. 1995) & Deletion of 249 amino acids & Deletion of TMS6-TMS11 \\
\hline prnB6 & - & - & $\mathrm{ATT}^{357} \rightarrow \mathrm{AAT}^{357}$ & PrnB-I119N & L2-TMS3 \\
\hline PrnB32 & - & - & $\mathrm{TGG}^{1328} \rightarrow \mathrm{TGA}^{1328}$ & PrnB-W409stop & L9-TMS10 \\
\hline prnB36 & - & - & TGG $^{1136} \rightarrow$ TGA $^{1136}$ & PrnB-W345stop & TMS8 \\
\hline prnB81 & - & - & TTC $^{935} \rightarrow$ GTC $^{935}$ & PrnB-F278V & L6-TMS7 \\
\hline prnB82 & - & - & Insertion $\mathrm{G}^{1093}$ & PrnB-Frameshiftstop (TAA ${ }^{1148}$ ) & TMS8 \\
\hline prnB206 & - & - & Duplication CCCTAT ${ }^{1247}$ & PrnB-PY382PYPY & L8-TMS9 \\
\hline prnB1110 & - & - & $\mathrm{TAT}^{959} \rightarrow \mathrm{TAA}^{959}$ & PrnB-Y286stop & TMS7 \\
\hline prnB144 & - & ++ & $\mathrm{GGG}^{360} \rightarrow \mathrm{TGG}^{360}$ & PrnB-G120W & L2 \\
\hline prnB411 & - & ++ & $\mathrm{ACC}^{1517} \rightarrow \mathrm{AAC}^{1517}$ & PrnB-T456N & L10-TMS11 \\
\hline prnB115 & $+(+)$ & ++ & Insertion $\mathrm{T}^{1801}$ & PrnB-Extended & C-term \\
\hline prnB117 & - & ++ & Duplication CCAACCGCA ${ }^{1233}$ & PrnB-NRT378NRTNRT & L8-TMS9 \\
\hline prnB119 & + & ++ & $\mathrm{TTT}^{845} \rightarrow \mathrm{TTG}^{845}$ & PrnB-F248L & TMS6 \\
\hline prnB508 & ++ & +++ & $\mathrm{GGA}^{1310} \rightarrow \mathrm{GAA}^{1310}$ & PrnB-G403E & L9-TMS10 \\
\hline
\end{tabular}

+++ define wild-type growth, - defines lack of growth typical of the non-utilization of a $\mathrm{N}$ source, and different number of + describe intermediate growth between +++ and - .

Mutations prnB-Q219R, prnB-Q219H, prnB-K245R, prnB$K 245 E$ and $p r n B-K 245 L$ were constructed and introduced by targeted homologous integration into the prnB genomic locus of $A$. nidulans, as described in Experimental procedures and previously in Tavoularis et al. (2001). Mutations prnB-Q219R and $p r n B-Q 219 H$ substitute the GIn found in proline transporters for two residues conserved in all other fungal amino acid transporters. Mutations prnB-K245R, prnB-K245E and prnB-K245L help define the role of the positive charge present in TMS6 of proline transporters. All mutant strains were analysed by growth tests for their ability to grow on proline or other nitrogen sources. Figure 4 shows that prnBQ219R, prnB-Q219H, prnB-K245L and prnB-K245R mutant strains grow similarly to a $p r n B^{+}$strain in media containing proline as the sole nitrogen source at both 37 and $25^{\circ} \mathrm{C}$. Mutant strain prnB-K245E showed a mildly reduced growth on proline at $25^{\circ} \mathrm{C}$ but normal growth at $37^{\circ} \mathrm{C}$. All mutants showed normal growth on all other nitrogen sources tested (results not shown).

\section{Transport properties of the prnB mutants}

A kinetic analysis was carried out of proline uptake in all mutant strains described in this work. All uptake experiments were performed at $37^{\circ} \mathrm{C}$. It should also be emphasized that $V$-values represent apparent capacities for proline transport, as they are directly dependent on the amount of PrnB present at the plasma membrane, a variable that one cannot

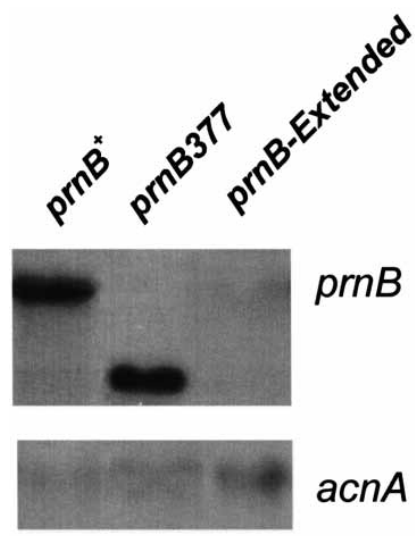

(a)

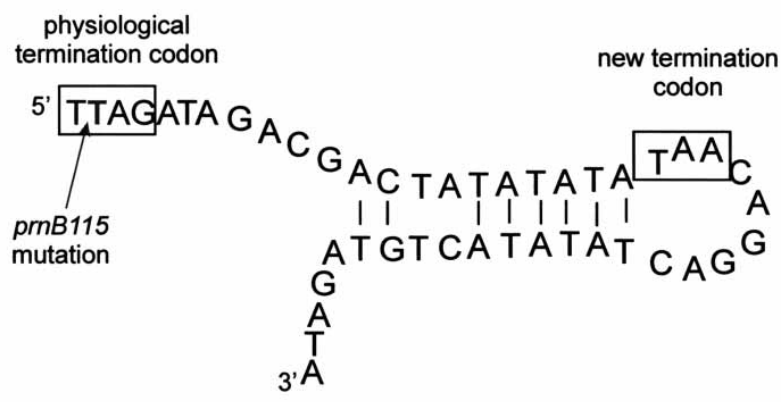

(b)

Figure 2. $\quad$ prnB mRNA steady state levels $(a)$ and model of prnB-Extended stem-loop structure $(b)$. (a) Northern blot analysis of $10 \mu \mathrm{g}$ total RNA extracted from mycelium grown at $25^{\circ} \mathrm{C}$ for $16 \mathrm{~h}$ under non-inducing conditions (urea as nitrogen source) followed by $4 \mathrm{~h}$ of growth in the presence of $20 \mathrm{mM} \mathrm{L-ornithine} \mathrm{(inducing} \mathrm{conditions} \mathrm{independent} \mathrm{of} \mathrm{PrnB} \mathrm{function;} \mathrm{ornithine} \mathrm{is} \mathrm{taken} \mathrm{up} \mathrm{by} \mathrm{a} \mathrm{transporter} \mathrm{other} \mathrm{than} \mathrm{PrnB} \mathrm{and} \mathrm{is}$ converted intracellularly to proline (Dzikowska et al. 1999). Therefore, prnB mutations do not affect induction by ornithine). RNA was extracted from a prnB ${ }^{+}$strain, a strain carrying a prnB deletion (prnB377) and the mutant strain prnB-Extended. RNA transferred onto nitrocellulose filters was hybridized with $p r n B$ - and acnA-specific probes, as described in Experimental procedures. acnA is the $A$. nidulans actin gene used as an internal control to monitor RNA amounts (Fidel et al. 1988). (b) PrnB-Extended stem-loop structure. Analysis of the 3' mRNA region of the prnBExtended allele using the DNA Strider programme (Marck 1988). The stop codons of the prnB ${ }^{+}$and the prnB-Extended alleles are shown within boxes. The possible interactions for the formation of a secondary structure are indicated by vertical lines. The selection of the region analysed is arbitrary. 


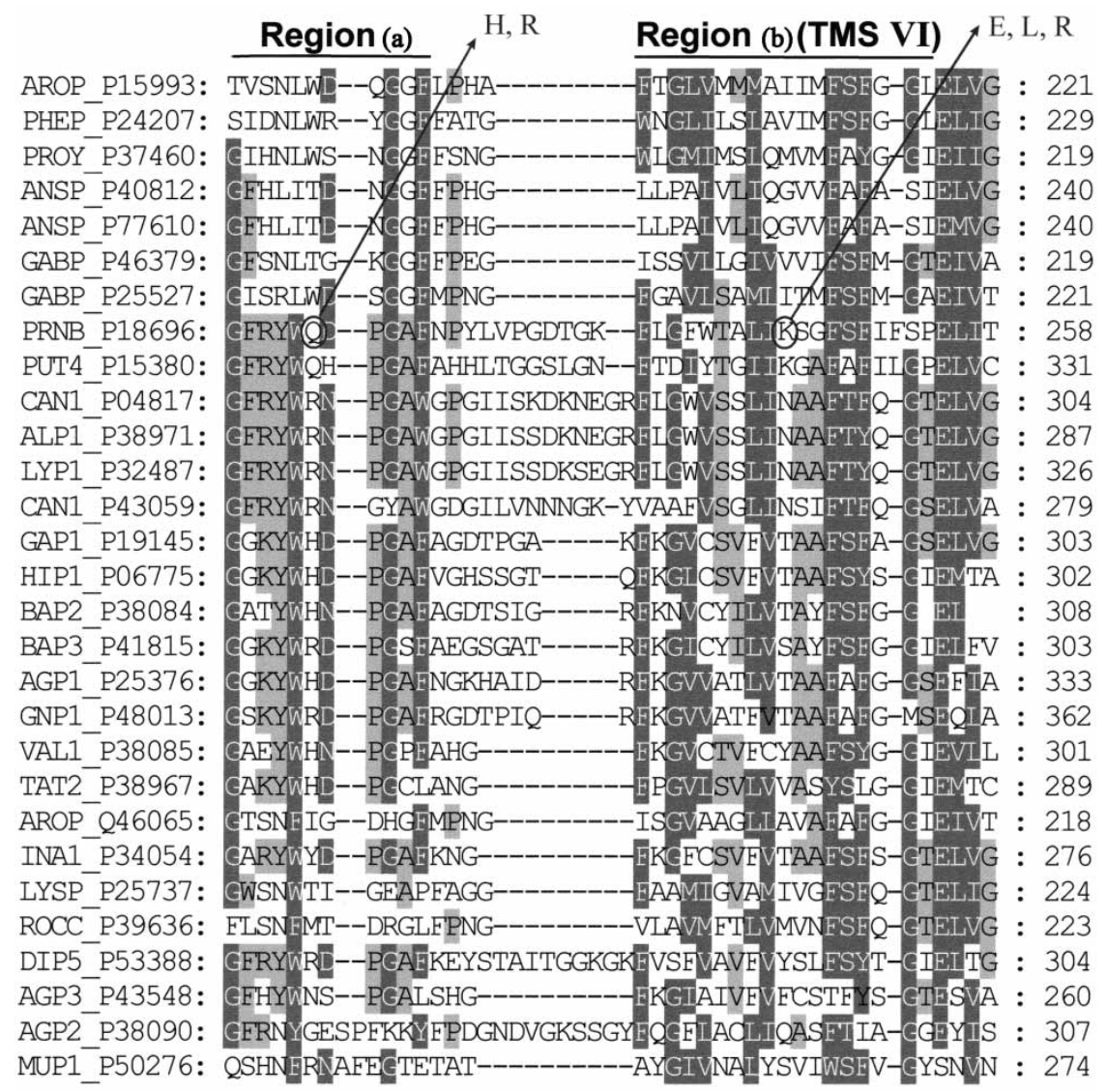

Figure 3. Sequence alignment of bacterial and fungal amino acid transporters. Two regions of high amino acid similarity, $(a)$ and $(b)$, are presented. Region (a) is located in L5 and region B in TMS6 in all amino acid transporters. Q219 and K245 (numbering refers to A. nidulans) conserved only in proline transporters of $A$. nidulans and $S$. cerevisiae map in regions $(a)$ and $(b)$, respectively. Similar amino acids are shown in dark and light grey boxes, according to the degree of similarity ( $>60 \%$ and $>40 \%$, respectively). Small dashes indicate gaps introduced by the programme to maximize similarity. AROP (P15993), PHEP (P24207), ANSP (P77610), GABP (P25527) and LYSP (P25737) are E. coli permeases, specific for aromatic amino acids, phenylalanine, asparagine, GABA and lysine, respectively. PROY (P37460) and ANSP (P40812) are Salmonella typhimurium permeases, specific for proline and asparagine, respectively. GABP (P46379) and ROCC (P39636) are B. subtilis permeases, specific for GABA and possibly all amino acids, respectively. AROP (Q46065) is a permease specific for aromatic amino acids from Corynebacterium glutamicum. INA1 (P34054) is a putative general amino acid permease from Trichoderma harzianum. PRNB is the A. nidulans proline-specific permease. CAN1 (P43059) is a permease-specific for the basic amino acids lysine and arginine from Candida albigans. PUT4 (P15380), CAN1 (P04817), ALP1 (P38971), LYP1 (P32487), HIP1 (P06775), BAP2 (P38084), BAP3 (P41815), AGP1 (P25376), GNP1 (P48813), VAL1 (P38085), TAT2 (P38967), DIP5 (P53388) and MUP1 (P50276) are S. cerevisiae permeases specific for proline, arginine, basic amino acids, lysine, histidine, leucine/valine/isoleucine, valine, asparagine/glutamine, glutamine, valine/tyrosine/tryptophan, tryptophan, glutamate/aspartate and methionine, respectively. Finally, GAP1 (P19145), AGP3 (P43548) and AGP2 (P38090) are general amino acid permeases from S. cerevisiae. In parentheses, the accession number of each protein in the SwissProt database is indicated. The directed mutations carried out in the present work are indicated by arrows.

estimate immunologically due to very low PrnB expression levels (Tavoularis et al. 2001).

In agreement with growth tests, mutants not growing on proline as a sole nitrogen source (nonsense or frameshift mutations and missense mutations prnB-I119N, prnB-F278V and prnB-PY382PYPY) show the same level of residual proline uptake as that found in a strain carrying a deletion of the prnB gene (results not shown). This is due to other minor amino acid transporter(s) able to incorporate proline and it never exceeds $20 \%$ of the total uptake (Arst et al. 1980, Tazebay et al. 1995, Scazzocchio C. and Apostolaki A., Personal communication). Table 2 summarizes the results obtained with all other mutations studied. All partial loss-offunction mutants obtained by selection for proline toxicity
(prnB-NRT378NRTNRT, prnB-Extended, prnB-F248L, prnB-G120W, prnB-T456N and prnB-G403E) have 1.5-5fold reduced apparent $V$-values, in agreement with their reduced growth on proline as a nitrogen source (see Figure 1). Among these mutations, only prnB-F248L also reduces the affinity of PrnB for proline significantly (4-4.5-fold). Substitutions of Q219 had a significant (6-fold) up-effect on the capacity of PrnB to transport proline and have no obvious growth phenotype on proline as a nitrogen source. Different substitutions of K245 had different effects on PrnB function. Mutant prnB-K245R showed proline uptake kinetics nearly identical to a $\mathrm{prnB}^{+}$strain. While both $\operatorname{prnB}-K 245 \mathrm{~L}$ and prnB-K245E mutants showed approximately 2-3-fold reduced capacity for proline transport, the former has a 


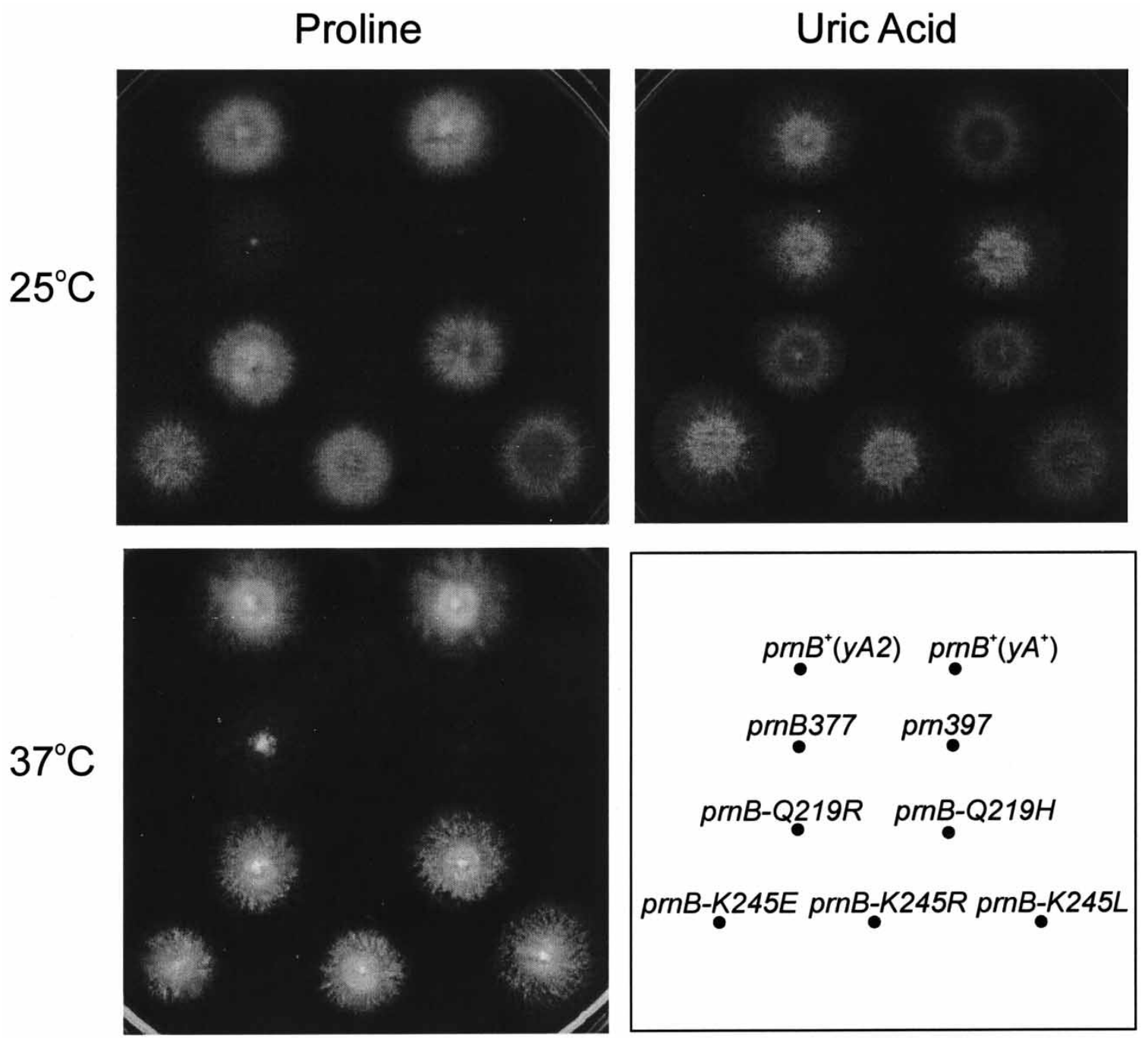

Figure 4. Growth of $A$. nidulans mutant strains. Growth of control strains (prnB ${ }^{+}$, prnB377), the recipient strain prn397 and the mutant strains (prnB-Q219R, prnB-Q219H, prnB-K245E, prnB-K245R and prnB-K245L) is shown. Because prnB mutations exist in either a green ( $\left.y A^{+}\right)$or yellow ( $y A 2$ ) conidiospore genetic background, the corresponding prnB $B^{+}$strains are shown. Growth was tested on $A$. nidulans $\mathrm{MM}$ supplemented with $5 \mathrm{mM}$ proline as sole nitrogen sources at 25 and $37^{\circ} \mathrm{C}$, as indicated. Strains grown on $595 \mu \mathrm{M}$ uric acid as a nitrogen source at $37^{\circ} \mathrm{C}$ are also shown. Growth tests were carried out for $60 \mathrm{~h}$ at $25^{\circ} \mathrm{C}$ or $48 \mathrm{~h}$ at $37^{\circ} \mathrm{C}$. Note the difference in growth between prnB397 (deletion of prnB and prnC) and prnB377 (deletion of prnB) strains, which allows recovery of even a complete loss-of-function mutation in prnB by the selection technique employed (see Experimental procedures).

significantly more reduced affinity for proline transport than the latter (8-fold compared to 2-fold). None of these changes affects growth of these mutants on proline (see Figure 4).

Interestingly, $p r n B-F 248 L$ and $p r n B-K 245 L$, the mutations affecting most (4- and 8-fold, respectively) the affinity of PrnB for its substrate, concern two amino acid residues on the same side of the TMS6 $\alpha$-helix (see below and Figure 6). F248 is an absolutely conserved residue in the APC family while K245 is conserved only in fungal proline transporters. One could speculate that such amino acids could directly or indirectly affect binding of substrates. Mutation prnB-F248L presented an additional interest due to work carried out in an homologous transporter. It has been shown that substitution of the homologous Phe residue for a Ser in Bap1p (branched chain amino acid transporter) and Hip1p (histidine transporter; Farcasanu et al. 1998) in S. cerevisiae led to transpor- ters with reduced amino acid uptake capacity, resistant to low $\mathrm{pH}$ and with the novel ability to take up $\mathrm{K}^{+}$ions (Wright et al. 1997). It was examined whether the mutation prnBF248L had a similar effect on PrnB function. Within the limits of growth tests carried out in the presence of different concentrations of $\mathrm{KCl}(10$ and $100 \mathrm{mM})$ and different $\mathrm{pHs}$ (4.8, 6.8 and 9.0) and uptake studies in different concentrations of $\mathrm{KCl}(10$ and $100 \mathrm{mM})$, one has been unable to demonstrate similar effects (results not shown). This might depend on which amino acid substitutes Phe.

One has also examined whether any of the mutations studied affect the absolute dependence, seen for the wildtype PrnB transporter (unpublished results), on a $\mathrm{H}^{+}$ gradient across the plasma membrane. In all PrnB mutants, similar to a wild-type strain, pre-incubation with $\mathrm{H}^{+}$-ATPase inhibitors $\left(\mathrm{N}^{\prime}, \mathrm{N}^{\prime}\right.$-dicyclohexylcarbodiimide) or protonophores 
Table 2. $\quad K_{\mathrm{m}}$ and $V$-values for ${ }^{3} \mathrm{H}$-proline uptake in the wild-type and in strains carrying PrnB mutations.

\begin{tabular}{lcc}
\hline Strains & $K_{\mathrm{m}}(\mu \mathrm{M})$ & $\left.\begin{array}{l}V_{\max }\left(\text { pmoles } \min ^{-1} 10^{8}\right. \\
\text { viable conidiospores }\end{array}{ }^{-1}\right)$ \\
\hline prnB ${ }^{+}$ & $34 \pm 2.0$ & $8.0 \pm 0.2$ \\
prnB-NRT378NRTNRT & $50 \pm 2.0$ & $3.2 \pm 0.05$ \\
PrnB-Extended & $30 \pm 2.0$ & $6.2 \pm 0.1$ \\
prnB-F248L & $134 \pm 10$ & $4.5 \pm 0.1$ \\
prnB-G120W & $33 \pm 2.0$ & $1.5 \pm 0.05$ \\
prnB-T456N & $35 \pm 2.0$ & $1.8 \pm 0.05$ \\
prnB-G403E & $71 \pm 3.0$ & $4.0 \pm 0.1$ \\
prnB-Q219R & $74 \pm 3.0$ & $52.8 \pm 3.0$ \\
prnB-Q219H & $58 \pm 3.0$ & $43.5 \pm 3.0$ \\
prnB-K245E & $61 \pm 3.0$ & $2.9 \pm 0.05$ \\
prnB-K245L & $281 \pm 10$ & $4.3 \pm 0.1$ \\
prnB-K245R & $34 \pm 2.0$ & $6.7 \pm 0.1$ \\
& &
\end{tabular}

Note: Proline uptake assays were performed as described in Experimental procedures. Proline uptake is expressed in pmoles per minute per $10^{8}$ viable conidiospores. The values shown represent averages of at least three independent assays showing no significant deviation.

(carbonyl cyanide $m$-chlorophenyl-hydrozone) abolished PrnB-dependent proline uptake (results not shown), indicating that $\mathrm{PrnB}$ is a proline/ $\mathrm{H}^{+}$symporter.

\section{Cellular expression of PrnB in selected mutant}

Results presented above clearly show that two mutations (K245L, F248L). affect the affinity $\left(K_{m}\right)$ of PrnB for proline. However, as far as it concerned the effect of different mutations on proline transport $(V)$, one could not draw any conclusion unless the amount of the protein in the plasma membrane was estimated. As has been discussed previously (Diallinas et al. 1998), the immunological detection of Aspergillus transporter proteins expressed from their native promoters has not been possible, and this is common also for many other transporters from different organisms, possibly due to low expression levels (Tavoularis et al. 2001 and references therein). On the other hand, over-expression of transporters often elicits a stress signal on the secretory pathway (unpublished observations) and induces the Unfolded Protein Response (Ma and Hendershot 2001). Thus, one has previously described the systematic use of functional PrnB-GFP chimeric molecules to study the cellular expression and topogenesis of PrnB from its native promoter (Tavoularis et al. 2001). Here, this system was used (see Figure 5 and Experimental procedures) to study selected prnB mutations, which affect proline uptake kinetics (K245L, K245E, F248L, T456N). Fluorometric quantification in cellfree crude extracts (see Experimental procedures and Tavoularis et al. 2001) showed that all mutants expressed $97-120 \%$ of the wild-type levels of GFP (results not shown). Figure 5(a) shows that all strains analysed carry a single copy of prnB-gfp integrated at the resident $p r n B$ locus, while Figure $5(b)$ shows the cellular expression of PrnB in the wildtype and the mutant strains, as seen by confocal laser microscopy. It is apparent that while mutations $\mathrm{K} 245 \mathrm{~L}$ and T456N affect partially the localization of PrnB in the plasma membrane, mutations K245E and F248L exhibit physiologi- cal cellular expression of PrnB and should, thus, directly affect PrnB function.

One has also investigated whether the presence of GFP affects the kinetics of different PrnB mutants. Similar to wildtype (Tavoularis et al. 2001), the presence of GFP connected with a four amino acid linker to the $\mathrm{C}$-terminus of PrnB does not affect the affinity $\left(K_{m}\right)$ of neither wild-type PrnB nor PrnB mutants for proline (results not shown). On the other hand, and as previously reported for the wild-type protein (Tavoularis et al. 2001), GFP has a negative effect on the capacity for proline transport, especially at $37^{\circ} \mathrm{C}$. $\mathrm{V}$-values in PrnB-GFP mutants are reduced to $50-75 \%$ of the values obtained with wild-type PrnB (results not shown). Thus, although GFP has not been an entirely silent tag, it has proved to be an excellent tool to investigate PrnB cellular expression in wild-type and prnB mutants.

\section{Implications of the topology of PrnB functional mutations}

Figure 6 shows the predicted topology of PrnB based on computer programmes (PRED-TMR algorithm, see Experimental procedures) utilizing multiply aligned APC transporters and experimental data from biochemical studies with homologous proteins, PheP (Pi and Pittard 1996, Cosgriff et al. 2000), AroA (Cosgriff and Pittard 1997, Cosgriff et al. 2000), LysP (Ellis et al. 1995), GabP (Hu and King 1998a) and Gap1p (Gilstring and Ljungdahl 2000). It must also be stressed that, using the same algorithms, none of the missense or duplication mutations described herein seem to alter significantly the hydrophobicity of any TMS. With the exception of a single mutation (G403A), all missense mutations affecting PrnB function map in the borders of cytoplasmic loops with transmembrane domains and in TMS6. The distribution of PrnB mutations to analogous findings was compared with other APC transporters. As already discussed above, it has been shown that specific mutations in TMS6 of Bap1p and Hip1p in S. cerevisiae led to transporters with altered substrate specificity (reduced amino acid uptake and the novel ability to take up $\mathrm{K}^{+}$ions). Altered substrate specificity mutations have also been identified in L1 (P113), L2-TMS3 (P148, V149, S152, Y173), L6 (G308, P313), L7 (Y356) and TMS10 (W451, $\mathrm{F} 461$ ) of the arginine transporter of $S$. cerevisiae (Can1p) and in TMS3 of the glutamine/asparagine transporter of $S$. cerevisiae Gnp1p (W239) (Regenberg and Kielland-Brandt 2001). In PheP (E. coli phenylalanine transport), it has been shown that mutations affecting function are located in the $\mathrm{N}$ terminal loop (R26, H27), L2-TMS3 (Y92, W95, F101, W105, E118), L4-TMS5 (E159, E161, K168), L6 (E226), TMS7 (R252) and L8 (R317). In addition, insertions of single Ala residues in L8-TMS9 had major effects on PheP transport activity ( $\mathrm{Pi}$ et al. 1998). In AroP (E. coli aromatic amino acid transport), site-directed mutagenesis has established that $a$ key residue involved in tryptophan transport is $\mathrm{Y} 103$ (Cosgriff et al. 2000). In other words, although mutations affecting the function of APC transporters map in various segments of different proteins, at least three regions seem of primary importance: L2-TMS3, TMS6 and L8-TMS9. The importance of L2-TMS3 is strengthened from the observation that, in all relevant studies, mutations affecting the function of amino 


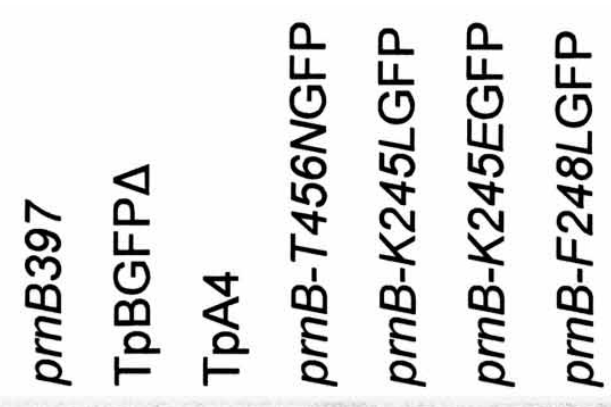

$-5-4.15 \mathrm{~kb}$

$-1.80 \mathrm{~kb}$

(a)
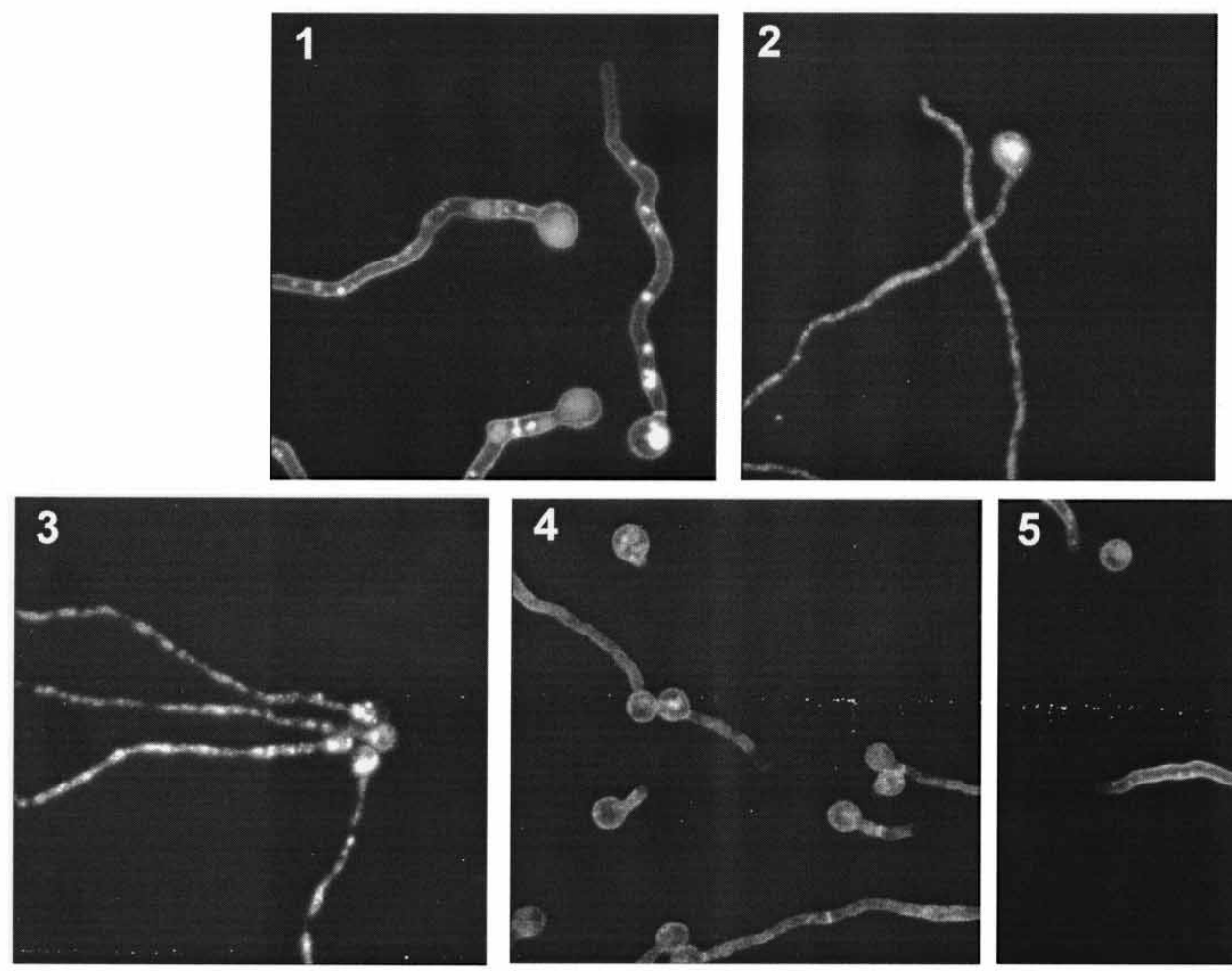

5

6

(b)

Figure 5 


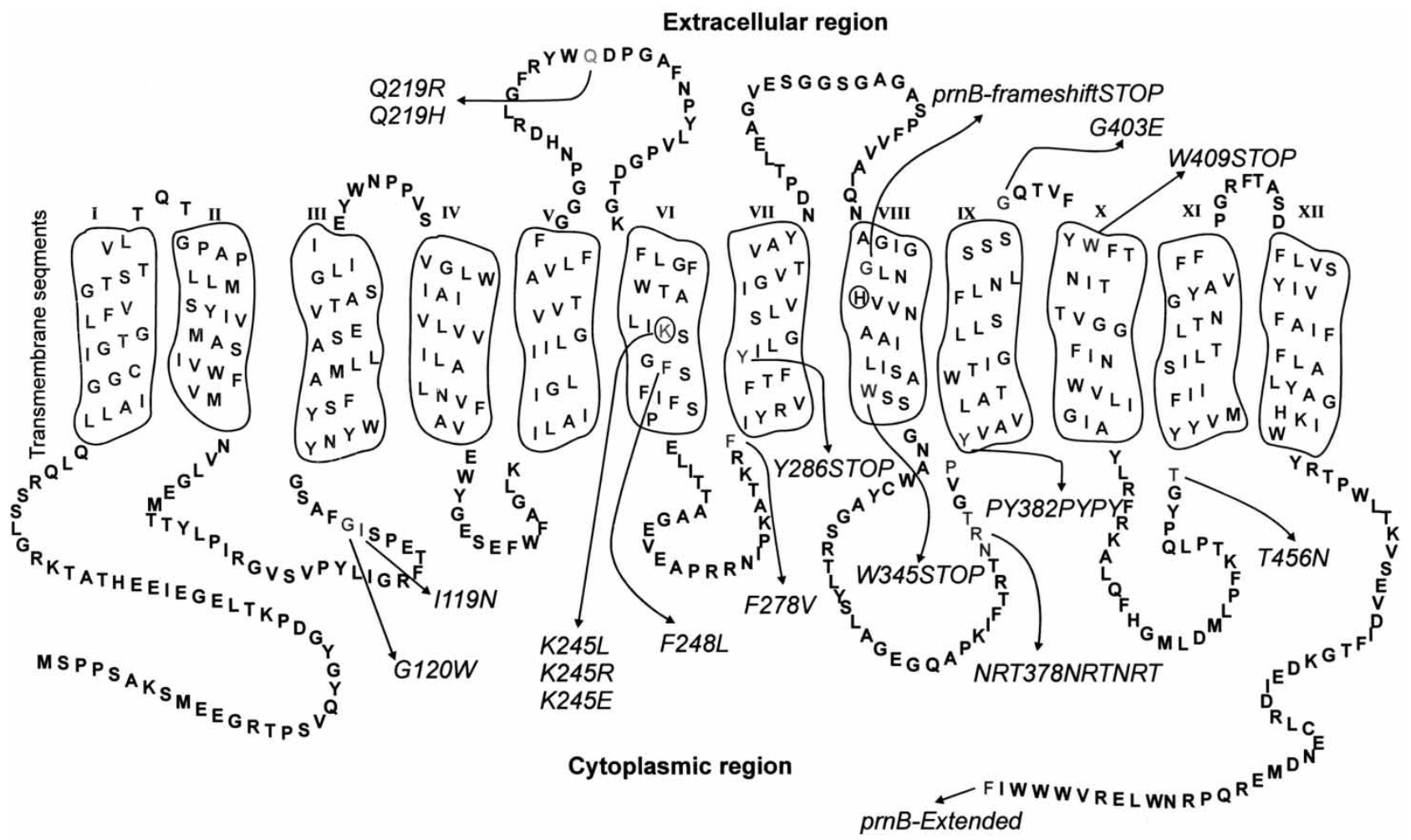

Figure 6. Predicted location of PrnB mutations. The PrnB polypeptide chain is shown crossing the membrane 12 times in a zigzag fashion with the $\mathrm{N}$ - and C-termini retained in the cytoplasm. This model has been drawn on the basis of the hydropathy blot and the 'positive-inside' rule (Von Heijne 1992), as described in Experimental procedures and the text. The transmembrane domains shown in boxes contain 19-22 amino acid residues. The two TMS charged residues (K245 and H334) are indicated by circles.

acid transporters were identified within this region. The role of TMS6 in substrate binding and transport is strongly supported by studies involving three different transporters, PrnB (herein), Bap1p and Hip1p (Wright et al. 1997). Finally, the importance of L8-TMS9 is underlined by the similar negative effects caused by insertions (or duplications) of amino acids in PheP and PrnB. Interestingly, this region corresponds to a consensus amphipathic region (CAR) shared by many APC transporters and the non-homologous mammalian GABA transporters active in the nervous system (Sophianopoulou and Diallinas 1995, Hu and King 1998b). The CAR has been also shown to be functionally significant in both GabP (Hu and King 1998a) and the mammalian mouse cationic amino acid transporters MCAT (Closs et al. 1993). The channel forming nature of the CAR suggests that it might be part of the substrate translocation pathway and that insertions or duplications of amino acid residues might directly affect its function or alter its topology relatively to other domains involved in amino acid binding and transport.

\section{Conclusions}

None of the previous studies addressing structure-function relationships in APC transporters has directly shown whether the mutations described have affected the cellular expression and translocation of transporters in the plasma membrane or the actual transport function of the protein. In a single study, a number of non-functional or altered specificity AroP-PheP chimeric proteins, expressed from high copy plasmids, have been studied using immunological detection with PheP-specific antisera (Cosgriff et al. 2000). This work studied mutations in an amino acid transporter expressed form its native promoter. It was shown that neither transcription nor translation are affected in different prnB mutants, as judged by northern blots and fluroscence quantification analyses of PrnB-GFP chimeras. It was also shown that mutant transporters still depend on a proton gradient across the plasma membrane. Using laser confocal microscopy, it was shown that mutations could be classified to two main groups: Those with partially defective topogenesis in the

Figure 5. Construction and analysis of isogenic strains carrying wild-type and mutant prnB-gfp genes. (a) Southern blot analysis of total genomic DNA extracted from the recipient strain prnB397, the control strains TpBGFP $\triangle$ and TpA4 and the mutant strains prnB-T456NGFP, prnB-K245LGFP, prnB-K245EGFP and prnB-F248LGFP (for strain details see Experimental procedures). 10 $\mu \mathrm{g}$ of genomic DNA from each strain were digested with $P s t$, transferred onto nitrocellulose filters and hybridized with a prnB-sgfp specific probe (as described in Experimental procedures and Tavoularis et al. 2001). The $\sim 4.15 \mathrm{~kb}$ seen in all transformants corresponds to the homologous integration of a single prnB-gfp chimeric gene in the prnB genomic locus (Tavoularis et al. 2001). Strain TpBGFP $\Delta$ yields the expected $\sim 1.8 \mathrm{~kb}$ band. Molecular weight markers are indicated in kb. (b) Representative photos from confocal laser microscopy of TpA4 (1) and the mutant strains, prnB-T456NGFP (2), prnBK245LGFP (3), prnB-K245EGFP (4) and prnB-F248LGFP (5), grown under induced conditions (20 mM proline-glucose) for $16 \mathrm{~h}$ at $25^{\circ} \mathrm{C}$. 
plasma membrane and those with no obvious cellular defect but reduced transport activity. Kinetic analysis of the latter clearly demonstrated that some mutants are directly affected in proline binding and transport (notice the lack of transport activity reflected in the lack of growth of PrnB-F248L on proline, despite an absolutely physiological cellular expression at $25^{\circ} \mathrm{C}$ ). The molecular and genetic tools described herein will allow one to investigate in detail aspects of APC transporters such as interactions with their substrates or interactions with chaperones involved in their controlled subcellular localization.

\section{Experimental procedures}

Media, growth conditions and strains for A. nidulans

Minimal (MM) and Complete (CM) media and growth conditions for A. nidulans have previously been described (Cove 1966). Supplements were added when necessary. Nitrogen sources, urea and proline were used at a final concentration of 5-10 mM. Uric acid was used at a concentration of $595 \mu \mathrm{M}$. The carbon sources, glucose and ethanol, were used at final concentrations of $1 \% \mathrm{w} / \mathrm{v}$ and $\mathrm{v} / \mathrm{v}$, respectively. The $A$. nidulans strains used have the following genotypes: $y A^{+}$pabaA1 $\left(p r n B^{+}\right), y A 2$ pantoB100 $\left(p r n B^{+}\right)$, argB2 biA1 pantoB100, yA2 pabaA1 prnB377 (prnB377), yA2 pabaA1 riboB2 pantoB100 prn397 (prnB397) and yA2 pabaA1 sasA60. prn397 is a deletion starting at the Pst l site of the prnB gene and extending up to the Pst I site of the prnC gene (Tavoularis et al. 2001). prnB377 is a deletion in the open reading frame of the prnB gene that was described previously in Tazebay et al. (1995). TpBGFP $\Delta$ and TpA4 strains were derived from prn397 strain transformed with plasmids $\mathrm{pBC} 9$ and $\mathrm{pA} 4$, respectively (Tavoularis et al. 2001). prnB-T456NGFP, prnB-K245LGFP, prnB-K245EGFP and prnB-F248LGFP strains were derived from prn397 transformed with plasmids pA4 carrying the corresponding mutant prnB alleles constructed by in vitro site-directed mutagenesis (Ex-Site PCRBased Site-Directed Mutagenesis Kit; Stratagene, La Jolla, CA). In all cases, the sequence of the entire prnB and gfp open reading frames was determined using the $A B I 310$ Genetic Analyser at the Institute of Biology, NCSRD, Athens, Greece. All other strains including prnB alleles isolated in this work were either selected from $y A 2$ pabaA1 sas $A 60$ strain or constructed in strain yA2 pabaA1 riboB2 pantoB100 prn397. pantoB100, pabaA1, riboB2, biA1 and $\arg B 2$ indicate auxotrophies for $\mathrm{D}$-pantothenic acid, $p$-aminobenzoic acid, riboflavine, biotin and arginine, respectively. $y A^{+}$indicates green conidia, while $y A 2$ results in yellow conidia. These markers do not affect the regulation of gene products involved in proline uptake and catabolism. The sasA60 mutation leads to toxicity of compounds, which can be converted to semi-aldehydes such as L-proline and GABA ( $\gamma$-amino-n-butyrate). The toxicity of L-proline to sasA60 strains is such that mutations conferring resistance can be selected (Arst et al. 1981).

\section{DNA manipulations and protoplast transformation}

Plasmid isolation from Escherichia coli strains and standard DNA manipulations were performed as previously described (Sambrook et al. 1989). Polymerase Chain Reaction (PCR) was carried out using AmpliTaq DNA polymerase (Perkin-Elmer) and the Expand High Fidelity PCR system (Roche Molecular Biochemicals, Mannheim, Germany). DNA sequencing of plasmid constructions and PCR products were carried out using the $A B I 310$ Genetic Analyser at the Institute of Biology (NCSRD, Athens, Greece). The oligonucleotide primers, specific for the prnB gene, used for sequencing and PCR amplifications were:

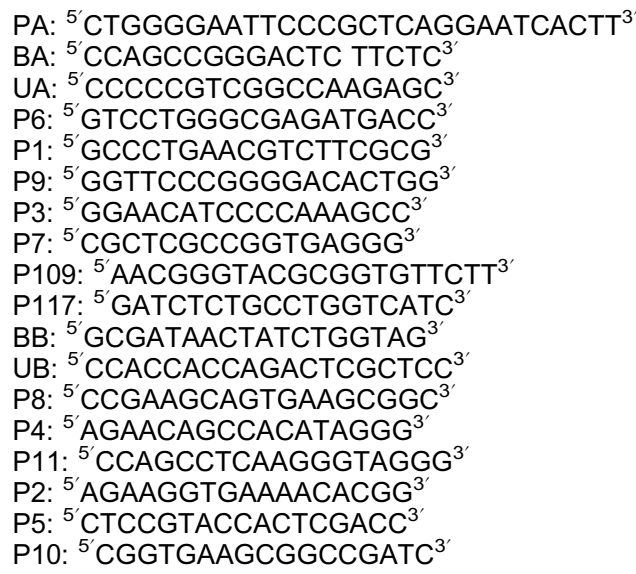

The oligonucleotide primers used for the in vitro construction of prnB alleles were:

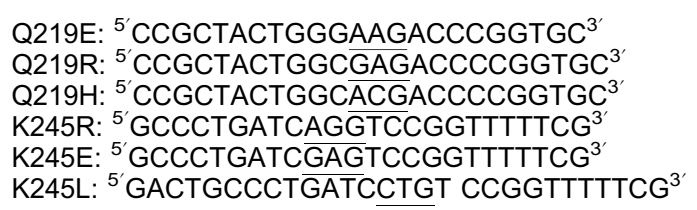

A. nidulans protoplast transformation was carried out as described by Tilburn et al. (1983). Total genomic DNA isolation from $A$. nidulans strains and Southern blot analysis has been carried out as in Lockington et al. (1985). The DNA fragment used as a probe in Southern blots was a $\sim 2 \mathrm{~kb}$ PpuMl-BamHI restriction fragment of plasmid pA4 (Tavoularis et al. 2001). Northern blot analysis has been carried out using the glyoxal method described by Tazebay et al. (1997). The DNA fragment used as a probe in northern blots was a $\sim 1.8 \mathrm{~kb}$ Pstl restriction fragment of the prnB gene (Sophianopoulou and Scazzocchio 1989) isolated from plasmid pAN225 (Hull et al. 1989, Tavoularis et al. 2001). The A. nidulans actin gene (acnA) is used as an internal control to monitor the amount of RNA loaded in different lanes (Fidel et al. 1988, Tazebay et al. 1997).

\section{Genetic isolation of prnB mutations}

Early genetic work had led to the isolation of several prnB-specific mutations (prnB6, prnB50, prnB32, prnB36, prnB81, prnB82, prnB109, prnB1110, prnB206) (Arst and MacDonald 1975, Arst et al. 1981, Jones et al. 1981, Arst Jr., H. N., Personal communication). Those used in the present work were selected either as spontaneous mutations conferring resistance to $50 \mathrm{mM} \mathrm{L}$-proline in a strain of genotype proA6 sasA60 (prnB81, prnB82) or proB9 prnD156 (prnB206), as described by Arst et al. (1981), or after treatment of a strain of genotype proA6 with $\mathrm{N}$-methyl- $\mathrm{N}^{\prime}$-nitro-Nnitrosoguanidine (NTG) (prnB6, prnB32 and prnB36), as described by Arst and MacDonald (1975). The toxicity of L-proline to sasA60 strains is such that mutations conferring resistance can be selected (see above, Arst et al. 1981). The proA6 mutation results in an Lproline requirement, which facilitates screening of prnB mutations, distinguishing them from mutations in other genes (Arst et al. 1980, Tazebay et al. 1995). This work modified the original genetic selection in order to isolate partial loss-of-function or conditional prnB mutations. prnB mutants were selected on an $A$. nidulans strain with a sas $A 60$ genetic background ( $y A 2$ pabaA1 sas $A 60$ ), as spontaneous or UV-induced sectors conferring resistance to $50 \mathrm{mM}$ L-proline, as described by Arst et al . (1981), at both 25 and $37^{\circ} \mathrm{C}$. All mutant strains were then tested at both temperatures, in order to recognize cryo- and temperature sensitive mutants. Selected putative prnB mutants were crossed to a strain argB2 biA1 pantoB100 to segregate out the sas $A 60$ mutation. 
Construction of targeted prnB alleles and prnB-gfp chimeric genes

In vitro prn $B$ directed mutagenesis was performed by the method of Kunkel et al. (1987) using plasmid pBHX1. This plasmid contains the EcoRI-Hind III fragment of plasmid pAN225 including the prnB gene (Tavoularis et al. 2001). The codon $C A A^{758}$ that specifies amino acid residue Q219 of PrnB was substituted for CAC $^{758}$ (mutation H219) and $\mathrm{CGA}^{758}$ (mutation R219). The codon $A A^{836}$ that specifies amino acid residue K245 of PrnB was substituted for GAG $^{836}$ (E245), $\mathrm{CTG}^{836}$ (L245) and $\mathrm{AGG}^{836}$ (R245). The fragments PpuMl-SphI $(\sim 1.2 \mathrm{~kb})$ of the plasmid pBHX1 containing the mutations were sequenced to verify that none contains any other mutations and were used to substitute for the same fragment of plasmids pBC9 or pA4. Plasmid pBC9 contains a $\sim 4.9 \mathrm{~kb}$ EcoRI-PflMl fragment, derived from plasmid pAN225, containing the prnB gene and part of the prnC gene in a bluescript $\mathrm{KS}(+)$ vector (Tavoularis et al. 2001). pA4 is a version of pBC9 in which the prnB open reading frame is fused in-frame with the gfp open reading frame via a specific four amino acid linker (Tavoularis et al. 2001). Upon transformation of the mutant strain prn397, which carries a large deletion extending from within the $p r n B$ open reading frame to within the prnC open reading frame, with linearized pBC9 or pA4 plasmids containing the mutations, transformants are directly isolated on proline as the sole nitrogen source. Strain prn397 lacks both PrnB and PrnC ( L- $\Delta^{1}-$ pyrroline-5-carboxylate dehydrogenase) activities and does not grow at all on media containing proline as a sole nitrogen source, as the absence of PrnC results in strong proline toxicity. This strain allows the direct selection of $\mathrm{prnC}^{+}$transformants upon reintroduction of sequences containing any $p r n B$ allele and the missing prnC sequences, notwithstanding whether or not the introduced prnB sequences are functional. This is because even complete loss-offunction mutant in prnB (see below) allows leaky growth on proline, which can easily be assessed on the background of the prnB-prnC deleted strain. The functionality of the PrnB protein can then be assessed directly by growth on proline as a sole nitrogen source (Tavoularis et al. 2001). This system leads to the targeted, singlecopy integration of any $p r n B$ allele or prnB-gfp chimeric gene at the resident prn $B$ locus, thus avoiding complications arising from ectopic and/or multiple integrations of prnB copies.

\section{Confocal laser microscopy}

Samples were prepared as described previously in Tavoularis et al. (2001). Confocal laser microscopy was carried out on a BIO-RAD MRC 1024 CONFOCAL SYSTEM, Laser Sharp Version 3.2 Bio-Rad Software, Zoom $\times 4$, Excitation/emission: 488nm/Blue, Samples at Laser Power $10 \%$, Kalman filter $n=5-6,0.3 \mu \mathrm{m}$ cut, Iris: $7-8$, Crypton/Argon Laser. Nikon DIAPHOT 300 Microscope, $\times 60$ (Oil immersion) Lens Emission Filter 522/DF35. Lens Reference: Plan Apo 60/1.40 oil DM (Nikon, Japan) 160175, 60 DM/Ph4, 160/0.17.

\section{Fluorometry}

Samples were prepared as described previously in Tavoularis et al. (2001). A Perkin Elmer Fluorescence Spectrophotometer MPF-3 was used. Emission was detected at $510 \mathrm{~nm}, \Delta \lambda=6 \mathrm{~nm}$ when 488 $\mathrm{nm}, \Delta \lambda=4 \mathrm{~nm}$ was used as the excitation wavelength. Values were normalized against the amount of total protein in the samples and expressed as relative GFP fluorescence units per micrograms of protein. Protein concentration was determined using a modified Bradford assay (Bradford 1976).

\section{Proline transport assays}

$\left[2,3,4,5-{ }^{3} \mathrm{H}\right] \mathrm{L}$-proline uptake was assayed in germinating conidia at $37^{\circ} \mathrm{C}$, as previously described (Tazebay et al. 1995, Meintanis et al. 2000). Standard uptake assays for the determination of initial uptake rates were performed in $A$. nidulans $\mathrm{MM}(\mathrm{pH} 6.5)$ by using $10 \mu \mathrm{M}$ $\left[2,3,4,5-{ }^{3} \mathrm{H}\right]$ L-proline (specific activity $120 \mathrm{Ci} \mathrm{mmol}^{-1}$; Moravek, Biochemicals, Brea CA, USA). Initial uptake rates were expressed in pmol of substrate incorporated per 1 min per $10^{8}$ viable conidia. Radioactivity was determined in sediment and supernatant by liquid scintillation counting (Beckman Instruments). Transport measurements were repeated independently and the reported results represent the mean values of at least three-to-five different experiments. The apparent Michaelis constant $\left(K_{\mathrm{m}}\right)$ and maximal velocity (V) values for ${ }^{3} \mathrm{H}$ L-proline were determined from double reciprocal plots of the initial uptake rates against substrate concentration. Initial uptake rates were corrected by subtracting background uptake values, evident in the prn377 total loss-of-function mutant strain, calculated in every uptake experiment (Tazebay et al. 1995). The errors given are standard deviations of the mean value.

\section{In silico analyses}

Sequences were collected from the SwissProt database using the programme BLASTP (Altschul et al. 1997) and were aligned using the programme CLUSTAL-X (Thompson et al. 1997). Predicted topology of PrnB based on computer programmes (PRED-TMR algorithm; http://biophysics.biol.uoa.gr/PRED-TMR; Pasquier et al. 1999) and experimental data from biochemical studies with homologous proteins.

\section{Acknowledgements}

This work was partially supported by the grant 'DEMOEREYNA' of NCSR Demokritos to V. S. and PLATON 70/3/5170 to C. S. and V. S. Work at Orsay was supported by the CNRS, the Universite ParisSud and the Institut Universitaire de France. Work in the lab of A. R. was supported by the International Programme of HHMI (USA). U. H. T. was supported by the Turkish Academy of Sciences Young Investigator Award (TÜBA-GEBIP/2001-2-18) and by Bilkent University Research Funds. We also thank Dr K. Stamatakis and Dr M. Sagnou for support.

\section{References}

Altschul, S. F., Madden, T. L., Schaffer, A. A., Zhang, Z., Miller, W. and Lipman, D. J., 1997, Gapped BLAST and PSI-BLAST: a new generation of protein database search programs. Nucleic Acids Research, 25, 3389-3402.

Arst Jr., H. N. and Cove, D., 1973, Nitrogen metabolite repression in Aspergillus nidulans. Molecular and General Genetics, 126, 111141.

Arst Jr., H. N. and MacDonald, D. W., 1975, A gene cluster in Aspergillus nidulans with an internally located cis-acting regulatory region. Nature, 254, 26-31.

Arst Jr., H. N., Jones, S. A. and Bailey, C. R., 1981, A method for the selection of deletion mutations in the L-proline catabolism gene cluster of Aspergillus nidulans. Genetic Research, 38, 171-195.

Arst Jr., H. N., MacDonald, D. W. and Jones, S. A., 1980, Regulation of proline transport in Aspergillus nidulans. Journal of General Microbiology, 116, 285-294.

Bernard, F. and André, B., 2001, Genetic analysis of the signalling pathway activated by external amino acids in Saccharomyces cerevisiae. Molecular Microbiology, 41, 489-502.

Bradford, M. M., 1976, A rapid and sensitive method for quantitation of micrograms quantities of protein utilizing the principle of protein-dye binding. Analytical Biochemistry, 72, 248-254.

Burkovski, A. and Kramer, R., 2002, Bacterial amino acid transport proteins: occurrence, functions and significance for biotechnological applications. Applied Microbiological Biotechnology, 58, 265-274.

Causton, H., Py, B., McLaren, R. S. and Higgins, C. F., 1994, mRNA degradation in Escherichia coli: a novel factor, which impedes the exoribonucleolytic activity of PNPase at stem-loop structures. Molecular Microbiology, 14, 731-741.

Closs, E. I., Lyons, C. R., Kelly, C. and Cunningham, J. M., 1993, Characterization of the third member of the MCAT family of cationic amino acid transporters. Identification of a domain that determines the transport properties of the MCAT proteins. Journal of Biological Chemistry, 268, 20796-20800. 
Cosgriff, A. J. and Pittard, A. J., 1997, A topological model for the general aromatic amino acid permease, AroP, of Escherichia coli. Journal of Bacteriology, 179, 3317-3323.

Cosgriff, A. J., Brasier, G., Pi, J., Dogovski, C., Sarsero, J. P. and Pittard, A. J., 2000, A study of AroP-PheP chimeric proteins and identification of a residue involved in tryptophan transport. Journal of Bacteriology, 182, 2207-2217.

Cove, D. J., 1966, The induction and repression of nitrate reductase in the fungus Aspergillus nidulans. Biochimica et Biophysica Acta, 113, 51-56.

Cubero, B., Gomez, D. and Scazzocchio, C., 2000, Metabolite repression and inducer exclusion in the proline utilization gene cluster of Aspergillus nidulans. Journal of Bacteriology, 182, 233-235.

Diallinas, G., Valdez, J., Sophianopoulou, V., Rosa, A. and Scazzocchio, C., 1998, Chimeric protein analysis reveals a region involved in function and specificity of purine transporters in the filamentous fungus Aspergillus nidulans conserved in bacteria, plants and metazoan homologues. EMBO Journal, 17, $3827-$ 3837.

Durrens, P., Green, P. M., Arst Jr., H. N. and Scazzocchio, C., 1986, Heterologous insertion of transforming DNA and generation of new deletions associated with transformation in Aspergillus nidulans. Molecular and General Genetics, 203, 544-549.

Dzikowska, A., Swianiewicz, M., Talarczyk, A., Wisniewska, M., Goras, M., Scazzocchio, C. and Weglenski, P., 1999, Cloning, characterisation and regulation of the ornithine transaminase (otaA) gene of Aspergillus nidulans. Current Genetics, 35, $118-126$.

Ellis, J., Carlin, A., Steffes, C., Wu, J. and Rosen, B. P., 1995, Topological analysis of the lysine-specific permease of Escherichia coli. Microbiology, 141, 1927-1935.

Farcasanu, I. C., Mizunuma, M., Hirata, D. and Miyakawa, T., 1998, Involvement of histidine permease (Hip1p) in manganese transport in Saccharomyces cerevisiae. Molecular and General Genetics, 259, 541-548.

Fidel, S., Doonan, J. H. and Morris, N. R., 1988, Aspergillus nidulans contains a single actin gene which has unique intron location and encodes a $\gamma$-actin. Gene, 70, 283-293.

Forsberg, H. and Ljungdahl, P. O., 2001, Genetic and biochemical analysis of the yeast plasma membrane Ssy1p-Ptr3p-Ssy5p sensor of extracellular amino acids. Molecular and Cellular Biology, 21, 814-826.

Gilstring, C. F. and Ljungdahl, P. O., 2000, A method for determining the in vivo topology of yeast polytopic membrane proteins demonstrates that Gap1p fully integrates into the membrane independently of Shr3p. Journal of Biological Chemistry, 275, 31488-31495.

Gonzalez, R., Gavrias, V., Gomez, D., Scazzocchio, C. and Cubero, B., 1997, The integration of nitrogen and carbon catabolite repression in Aspergillus nidulans requires the GATA factor AreA and an additional positive-acting element, ADA. EMBO Journal, 16, 2937-2944.

$\mathrm{Hu}, \mathrm{L}$. A. and King, S. C., 1998a, Functional sensitivity of polar surfaces on transmembrane helix 8 and cytoplasmic loop 8-9 of the Escherichia coli GABA (4-aminobutyrate) transporter encoded by gabP: mutagenic analysis of a consensus amphipathic region found in transporters from bacteria to mammals. Biochemistry Journal, 330, 771-776.

$\mathrm{Hu}, \mathrm{L}$. A. and King, S. C., 1998b, Membrane topology of the Escherichia coli gamma-aminobutyrate transporter: implications on the topography and mechanism of prokaryotic and eukaryotic transporters from the APC superfamily. Biochemistry Journal, 336, 69-76.

Hull, E. P., Green, P. M., Arst Jr., H. N. and Scazzocchio, C., 1989, Cloning and physical characterization of the L-proline catabolism gene cluster of Aspergillus nidulans. Molecular Microbiology, 3, $553-559$.

Jones, S. A., Arst Jr., H. N. and MacDonald, D. W., 1981, Gene roles in the prn cluster of Aspergillus nidulans. Current Genetics, 3 , $49-56$.
Kunkel, T. A., Roberts, J. D. and Zakour, R. A., 1987, Rapid and efficient site-specific mutagenesis without phenotypic selection. Methods in Enzymology, 154, 367-382.

Lockington, R. A., Sealy-Lewis, H. N., Scazzocchio, C. and Davies, R. W., 1985, Cloning and characterization of the ethanol utilisation regulon in Aspergillus nidulans. Gene, 33, 137-149.

Ma, Y. and Hendershot, L. M., 2001, The unfolding tale of the unfolded protein response. Cell, 107, 827-830.

Marck, C., 1988, DNA Strider': a 'C' program for the fast analysis of DNA and protein sequences on the Apple Macintosh family of computers. Nucleic Acids Research, 16, 1829-1836.

Martinez, P. and Ljungdahl, P. O., 2000, The SHR3 homologue from $S$. pombe demonstrates a conserved function of ER packaging chaperones. Journal of Cell Science, 23, 4351-4362.

Meintanis, Ch., Karagouni, A. D. and Diallinas, G., 2000, Amino acid residues N450 and Q449 are critical for the uptake capacity and specificity of UapA, a prototype of a nucleobase-ascorbate transporter family. Molecular Membrane Biology, 17, 47-57.

Pasquier, C., Promponas, V. J., Palaios, G. A. and Hamodrakas, S. J., 1999, A novel method for predicting transmembrane segments in proteins based on statistical analysis of the SwisProt database: the PRED-TMR algorithm. Protein Engineering, 12, 381-385.

$\mathrm{Pi}, \mathrm{J}$. and Pittard, A. J., 1996, Topology of the phenylalanine-specific permease of Escherichia coli. Journal of Bacteriology, 178, 2650-2655.

Pi, J., Dogovski, C. and Pittard, A. J., 1998, Functional consequences of changing proline residues in the phenylalaninespecific permease of Escherichia coli. Journal of Bacteriology, $180,5515-5519$.

Pi, J., Wookey, P. J. and Pittard, A. J., 1993, Site-directed mutagenesis reveals the importance of conserved charged residues for the transport activity of the PheP permease of Escherichia coli. Journal of Bacteriology, 175, 7500-7504.

Platt, A., Langdon, T., Arst Jr., H. N., Kirk, D., Tollervey, D., Sanchez, J. M. and Caddick, M. X., 1996, Nitrogen metabolite signalling involves the C-terminus and the GATA domain of the Aspergillus transcription factor AREA and the $3^{\prime}$ untranslated region of its mRNA. EMBO Journal, 15, 2791-2801.

Regenberg, B. and Kielland-Brandt, M. C., 2001, Amino acid residues important for substrate specificity of the amino acid permeases Can1p and Gnp1p in Saccharomyces cerevisiae. Yeast, 18, 1429-1440.

Reizer, J., Finley, K., Kakuda, D., MacLeod, C. L., Reizer, A. and Saier Jr., M. H., 1993, Mammalian integral membrane receptors are homologous to facilitators and antiporters of yeast, fungi, and eubacteria. Protein Science, 2, 20-30.

Saier Jr., M. H., 2000, Families of transmembrane transporters selective for amino acids and their derivatives. Microbiology, 146, $1775-1795$.

Sambrook, J., Fritsh, E. F. and Maniatis, T., 1989, Molecular Cloning: A Laboratory Manual (Cold Spring Harbor, NY: Cold Spring Harbor Laboratory).

Sophianopoulou, V. and Diallinas, G., 1995, Amino acid transporters of lower eukaryotes: regulation, structure and topogenesis. FEMS Microbiology Reviews, 16, 53-75.

Sophianopoulou, V. and Scazzocchio, C., 1989, The proline transport protein of Aspergillus nidulans is very similar to amino-acid transporters of Saccharomyces cerevisiae. Molecular Microbiology, 3, 705-714.

Sophianopoulou, V., Suarez, T., Diallinas, G. and Scazzocchio, C., 1993, Operator derepressed mutations in the proline utilization gene cluster of Aspergillus nidulans. Molecular and General Genetics, 236, 209-213.

Tavoularis, St., Scazzocchio, C. and Sophianopoulou, V., 2001, Functional expression of a green fluorescent protein-tagged proline transporter in Aspergillus nidulans. Fungal Genetics and Biology, 33, 115-125.

Tazebay, U. H., Sophianopoulou, V., Cubero, B., Scazzocchio, C. and Diallinas, G., 1995, Post-transcriptional control and kinetic characterization of proline transport in germinating conidiospores of Aspergillus nidulans. FEMS Microbiology Letters, 132, 27-37.

Tazebay, U. H., Sophianopoulou, V., Scazzocchio, C. and Diallinas, G., 1997, The gene encoding the major proline transporter of 
Aspergillus nidulans is upregulated during conidiospore germination and in response to proline induction and amino acid starvation. Molecular Microbiology, 24, 105-117.

Thompson, J. D., Gibson, T. J., Plewniak, F., Jeanmougin, F. and Higgins, D. G., 1997, The CLUSTAL-X windows interface: flexible strategies for multiple sequence alignment aided by quality analysis tools. Nucleic Acids Research, 25, 4876-4882.

Tilburn, J., Scazzocchio, C., Taylor, G. G., Zabicky-Zissman, J. H., Lockington, R. A. and Davis, R. W., 1983, Transformation by integration in Aspergillus nidulans. Gene, 26, 205-221.

Von Heijne, G., 1992, Membrane protein structure prediction. Hydrophobicity analysis and the positive-inside rule. Journal of Molecular Biology, 225, 487-494.
Wang, H., Kavanaugh, M. P., North, R. A. and Kabat, D., 1991, Cellsurface receptor for ecotropic murine retroviruses is a basic amino-acid transporter. Nature, 352, 729-731.

Wright, M. B., Ramos, J., Gomez, J. M., Moulder, K., Scherrer, M., Munson, G. and Gaber, R. F., 1997, Potassium transport by amino acid permeases in Saccharomyces cerevisiae. Journal of Biological Chemistry, 272, 13647-13652.

Received 14 October 2002; and in revised form 20 February 2003. 